\title{
Uso de metacaolín, vidrio reciclado y fibra óptica en la elaboración de un concreto translúcido
}

\section{Use of metakaolin, wasteglass and optical fiber in the development oftranslucent concrete}

\author{
Diana Marcela Franco Durán \\ Ingeniera Civil, Investigador Grupo INME \\ Universidad Industrial de Santander, Colombia. \\ dianita_franco8@hotmail.com
}

\author{
Edwin Pérez Sánchez \\ Ingeniero Civil, Investigador Grupo INME \\ Universidad Industrial de Santander, Colombia. \\ edwper@yahoo.es
}

\author{
Ricardo Alfredo Cruz Hernández \\ Ph.D., Ciencias Técnicas, Docente, Director Grupo INME \\ Universidad Industrial de Santander, Colombia. \\ racruz@uis.edu.co
}

\begin{abstract}
Resumen- Los materiales utilizados en la construcción están día a día en constante evolución y transformación. En su diseño se emplean diferentes materias primas y agregados, que pretenden mejorar sus propiedades físicas, químicas y de durabilidad. Asimismo, se busca que estos nuevos materiales sean una alternativa novedosa a nivel arquitectónico, disminuyan los costos y contribuyan con el medio ambiente. Se propone la creación de un concreto no estructural, modificado con metacaolín y que cuenta con características de translucidez, mediante la adición de vidrio reciclado y fibra óptica. EI comportamiento físico-mecánico del material evaluado, a partir de los ensayos de compresión, flexión y tracción, presentó una reducción considerable en su resistencia, debido a la inclusión de fibras ópticas. La adición de una sustancia puzolánica como el metacaolín (MK) al cemento mejoró la resistencia a la reacción álcali-sílice, que comúnmente se presenta por el alto contenido de sílice reactiva del vidrio, contribuyendo a la durabilidad del concreto no estructural. La traslucidez fue medida a partir de los ensayos de transmitancia y espectrofotometría, dando como resultado un material traslúcido. La cantidad de luz que atraviesa los especímenes es suficiente para que el ojo humano la capte.
\end{abstract}

Palabras clave—Concreto, Fibra óptica, Reacción álcali-sílice, Traslucidez, Vidrio.

Abstract- Nowadays, construction materials are in constantly development. They include different raw and aggregates to enhance its physical, chemical and durability properties. Likewise, these new materials should be a remarkable alternative, decreasing the costs and contributing with the environmental concerns. Our research proposes a nonstructural concrete modified with metakaolin(MK), which has translucency features thanksto addition of waste glass and optical fibers.The mechanical and physical behavior of the material was assessed by means of compression strength, flexural and tensile tests. The results indicatedthat each resistance (flexural, compression strength and tensile) significantlydecreasebecause ofaddition ofoptical fibers in the concrete. Besides, the pozzolanic material -metakaolin- added to the concreteimproved the alkali-silica reactionresistance,reducing the expansion producedin thematerialdue tothe high active silica content. Clearly, metakaolin helps to improvethe durability of the concrete. Translucency was measured from transmittance and spectrophotometry tests. As a result, the amount of light thatpass through the samples is enough to beseen by the human eye.

Keywords- Concrete, OpticalFiber, Alcali-SilicaReaction, Translucence, Glass.

\section{INTRODUCCIÓN}

En épocas recientes, el criterio de la construcción sustentable ha alcanzado una importancia relevante, así como interés por disponer de materiales con mejores propiedades y características especiales, como en este caso, contar con un concreto que permita ver a través de él.

Con el uso de residuos de vidrio, material con alta disponibilidad, se hace un aporte en la disminución de la contaminación generada por su inadecuada disposición final. Concretos base residuos de vidrio se han desarrollado por décadas [1],[2].

Estudios realizados indican que el vidrio por ser un material de naturaleza puzolánica, puede ser utilizado como un sustituto del cemento Portland [2]. Sin embargo, su uso como agregado produce una expansión considerable en la mezcla de concreto, debido a la reacción álcali-sílice que se presenta [3]. Con el fin de contrarrestar esta reacción, Ramlochan et al, (2000) demostraron que es posible controlarla, 
bajo la adición de metacaolín (MK) en proporciones de 10-15\% [4]. Por otra parte, se ha demostrado que la fibra óptica puede ser agregada al concreto, para permitir la transmisión de la luz visible [5].

Si bien estas investigaciones exponen que cada uno de estos materiales aporta diferentes propiedades y características al concreto, se han enfocado básicamente en éstos por separado.

Con este trabajo se propone la creación de un concreto no estructural modificado con metacaolín y que cuenta con características de translucidez, mediante la adición de vidrio reciclado y fibra óptica. Se busca además, un material que interactúe y contribuya con el medio ambiente y cumpla con los requisitos mínimos de resistencia, dureza y transmisión de la luz.

\section{PROGRAMA EXPERIMENTAL}

\subsection{Materiales}

El diseño de la mezcla se realizó con la intención de obtener un concreto traslúcido, de resistencia 21 Megapascales (MPa). Se utilizó un cemento hidráulico blanco (Cemento Portland Blanco Tipo I) y una relación agua-cemento $(\mathrm{a} / \mathrm{c})$ de 0.58 . Algunas de las características principales del cemento se indican en la Tabla I.

TABLA I

CARACTERÍSTICAS DEL CEMENTO PORTLAND BLANCO TIPO I

\begin{tabular}{|l|c|}
\hline \% Óxidos & Por Bolsa \\
\hline De Sílice $\left(\mathrm{SiO}_{2}\right)$ & 22.4 \\
De Aluminio $\left(\mathrm{Al}_{2} \mathrm{O}_{3}\right)$ & 5.04 \\
De Hierro $\left(\mathrm{Fe}_{2} \mathrm{O}_{3}\right)$ & 0.20 \\
De Calcio $(\mathrm{CaO})$ & 67.2 \\
De Magnesio $(\mathrm{MgO})$ & 1.30 \\
\hline Peso Específico [gr/cm $\left.{ }^{3}\right]$ & 3.14 \\
\hline Tiempo de Fraguado [h] & 1.00 \\
\hline Consistencia Normal del Cemento [\%] & 28.8 \\
\hline
\end{tabular}

La dosificación del concreto modificado se presenta en la Tabla II.

TABLA II

DOSIFICACIÓN DE LA MEZCLA DE CONCRETO PARA UN VOLUMEN DE $0.0025 \mathrm{~m}^{3}$

\begin{tabular}{|l|l|l|}
\hline MATERIAL & VOLUMEN NETO [m3] & PESO [Kg] \\
\hline Cemento & 0.0003 & 1.2 \\
\hline Agregado fino & 0.0009 & 2.7 \\
\hline Agregado grueso & 0.0005 & 1.3 \\
\hline Agua & 0.0006 & 0.6 \\
\hline Aire atrapado & 0.01 & 0 \\
\hline
\end{tabular}

Se remplazó alrededor de un $83.3 \%$ del total de los agregados fino y grueso por vidrio reciclado, obtenido de diferentes clases y tonalidades, con peso específico de $2.5 \mathrm{~g} / \mathrm{cm} 3$. El tamaño del vidrio utilizado como agregado grueso se encuentra entre el rango de tamices que pasan el No. 4 y son retenidos por el No. 8. El vidrio usado como agregado fino corresponde a tamaños que pasan el tamiz No. 8 hasta los retenidos por el No. 100.

Se utilizó caolín, procedente de Arcabuco, Boyacá, el cual fue pre-activado mediante su calcinación, a una temperatura aproximada de $800^{\circ} \mathrm{C}$, durante un periodo de dos horas. Este proceso, permitió la obtención de metacaolín (MK). Se remplazó cemento por metacaolín (MK) en proporción del $15 \%$ de su peso. Su adición en el concreto se realizó para mitigar la reacción álcali-sílice, producto del contenido de sílice reactiva presente en el vidrio ( $\mathrm{CaO} 12.9 \%, \mathrm{SiO}_{2} 74.4 \%, \mathrm{Na}_{2} \mathrm{O}$ 10.3\%) [6][7].

La fibra utilizada es un filamento de vidrio bastante delgado y flexible (de 2 a 125 micrones), capaz de conducir rayos ópticos (señales en base a la transmisión de luz). La fibra óptica se adicionó en un $5 \%$ del peso total de la mezcla. En la Tabla III se presentan las dosificaciones respectivas para cada uno de estos sustitutos.

TABLA III

DOSIFICACIÓN DEL VIDRIO, METACAOLÍN Y FIBRA ÓPTICA

\begin{tabular}{|l|c|c|}
\hline Sustituto & Porcentaje [\%] & Cantidad [gr] \\
\hline Vidrio & 83.3 & 3332 \\
\hline Metacaolín & 15 & 180 \\
\hline Fibra Óptica & 5 & 290 \\
\hline
\end{tabular}

Adicionalmente la Tabla IV indica la dosificación de un concreto (con una relación agua-cemento (a/c) de 0.65) caracterizado por Arciniegas y Fonseca, (2004) [8], denominado probeta de comparación. Los autores remplazaron parte del agregado fino por vidrio molido en proporciones del 10, 20 y $30 \%$ del volumen total de la mezcla. Esta probeta se utilizó para contrastar los resultados obtenidos de los ensayos de compresión, flexión y tracción.

TABLA IV

DOSIFICACIÓN DE LA PROBETA DE COMPARACIÓN

\begin{tabular}{|l|l|l|}
\hline MATERIAL & VOLUMEN NETO $[\mathrm{m} 3]$ & PESO $[\mathrm{Kg}]$ \\
\hline Cemento & 466.55 & 0.16 \\
\hline Agua & 303.26 & 0.30 \\
\hline Arena & 1399.65 & 0.33 \\
\hline
\end{tabular}




\subsection{Métodos}

Se realizaron ensayos para caracterizar el concreto translucido en su comportamiento físico-mecánico, durabilidad y transmisión de la luz. Se ensayaron concretos con y sin contenido de fibras ópticas, para evaluar su resistencia a la compresión, tracción y flexión a los 7,14 y 28 días.

Las muestras utilizadas en los diferentes ensayos, designadas como M1, M2 y M3 no contienen metacaolín (MK), mientras que, las designadas como M4, M5 y M6 incluyen metacaolín (MK) (15\% del peso del cemento).

Para el ensayo de compresión, se utilizaron probetas de forma cúbica, de $5 \mathrm{~cm}$ de lado, fabricadas conforme a la norma NTC220 [9] y ensayadas en la Máquina Universal de Ensayos Trebel, como se muestra en la Fig. 1a.

En el ensayo a flexión se emplearon probetas de sección cuadrada $4 \times 4 \mathrm{~cm}$ y de $16 \mathrm{~cm}$ de longitud (Ver Fig. 1b), fabricadas de acuerdo a la norma NTC 120 [10] y ensayadas en la Máquina Universal de Ensayos Trebel.

La caracterización de la resistencia a tracción del concreto, se determinó según la norma NTC 119
[11]. Las probetas se ensayaron en la Máquina Italiana de Ensayos de Mortero a Tracción (Officine Galileo Di Milano). Un esquema de su montaje se presenta en la Fig. 1c.

Estos ensayos se realizaron para muestras con y sin contenido de fibra ópticas ( $5 \%$ del peso del cemento). Los resultados obtenidos se compararon con los valores de la probeta de comparación caracterizada por [8].

Para evaluar la porosidad del concreto, se fabricaron 3 probetas cúbicas de $5 \mathrm{~cm}$ de lado, sometidas a un curado de 28 días. De cada una de ellas, se cortó una muestra cúbica de $2 \mathrm{~cm}$ de lado, descartando su parte superior e inferior y se escogieron aquellas con la forma más regular posible. Para esta prueba se utilizó el Porosímetro de Campo Ruska mostrado en la Fig. 2, y la porosidad del material se obtuvo a partir de la ley de Boyle, representada en la ecuación (1).

$$
P(\%)=\frac{(V t-V g)}{V t} * 100
$$

Donde $P$ es la porosidad, Vt es el volumen total, Vg es el volumen de los granos del especimen.

Fig. 1. MONTAJES DE LOS ENSAYOS A COMPRESIÓN (A), FLEXION (B) Y TRACCIÓN (C)

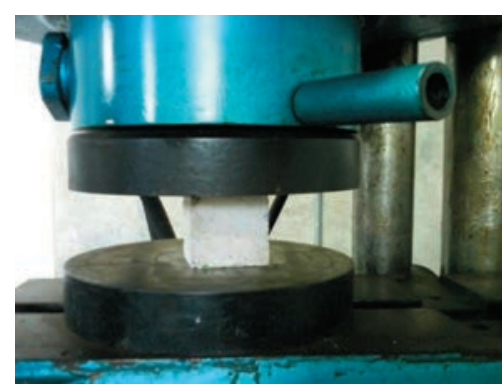

A

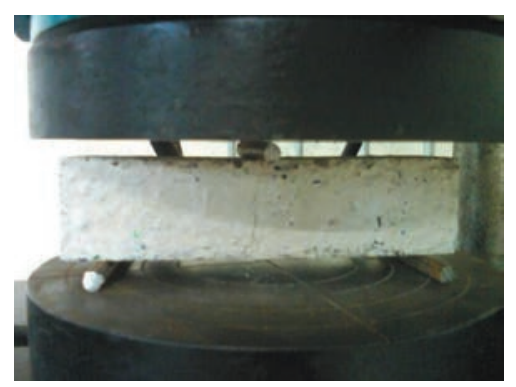

B

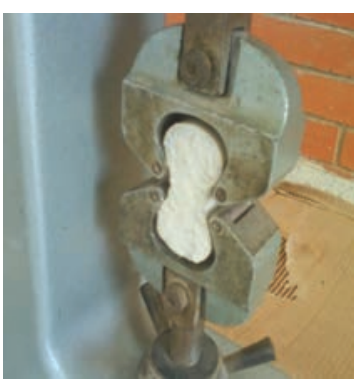

C

Fuente: Autores

Fig. 2. POROSÍMETRO DE CAMPO RUSKA Y PROBETAS CÚBICAS
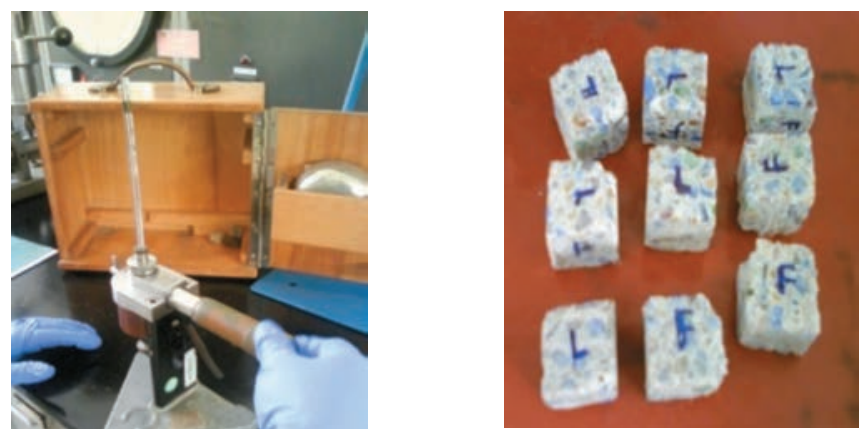

Fuente: Autores 
La permeabilidad se obtuvo utilizando probetas con características similares a las anteriores, mediante el Permeámetro de Gas Ruska. Este valor se calculó a partir del caudal de gas que pasa a través del núcleo, el gradiente de presión, la viscosidad del gas y las dimensiones de la probeta. A través del especimen, se hizo pasar gas Nitrógeno de Viscosidad $\mu=0.0176$ centipoises (Cp) a una temperatura de $23^{\circ} \mathrm{C}$ en dirección paralela y perpendicular a las fibras ópticas. La permeabilidad determinada es absoluta y se calculó mediante la siguiente ecuación (2).

$$
\mathrm{k}=\frac{\mu Q 1}{A \Delta P}
$$

Donde $\mathrm{k}$ es la permeabilidad, $\mu$ es la viscosidad del gas, $Q$ es el caudal del gas que pasa a través del material, A es el área transversal expuesta al flujo de gas y $\Delta \mathrm{P}$ es el gradiente de presión incidido a la muestra.

El ensayo de absorción capilar se desarrolló seguida la norma de ensayo Swiss Standard - SIA 162/1 [12]. Esta prueba se realizó con el fin de evaluar la reactividad del vidrio reciclado con los álcalis del cemento y el efecto de mitigación que produce la adición de la puzolana obtenida del caolín (Metakaolín).

Se elaboraron 4 probetas cúbicas de $5 \mathrm{~cm}$ de lado, sometidas a un curado de 28 días. A cada probeta se le hizo un corte de 5 milímetros en cada uno de sus extremos y se sumergieron hasta $3 \mathrm{~mm}$ de altura en agua. Las muestras M1 y M2 se colocaron con la superficie frontal en contacto con el agua (sentido paralelo a las fibras ópticas) y las muestras M3 y M4 con la superficie lateral en contacto con el agua (sentido perpendicular a las fibras ópticas). El peso de agua absorbida por unidad de superficie (W/A) en el tiempo t, se determinó a partir de las leyes de Darcy y de Laplace, mediante la ecuación (3).

$$
\frac{W}{A}=S * \sqrt{t}
$$

S es la capacidad de absorción capilar.

El ensayo para la determinación de la reactividad de los agregados (Vidrio), respecto a los álcalis del cemento y mitigación de la reacción álcali-sílice, se realizó seguidas las especificaciones descritas por la norma ASTM C1260-07[13]. En este ensayo se utilizaron 6 barras de mortero con vidrio reciclado, según la norma ASTM C490/C [14]. Estas barras fueron expuestas a una solución $1 \mathrm{~N}$ de $\mathrm{NaOH}$ a 80 $\pm 2{ }^{\circ} \mathrm{C}$, durante un periodo de 16 días, tiempo en el cual se hicieron mediciones diarias, para observar la expansión que se producía en las muestras.

Se evaluó la reactividad de las muestras con y sin contenido de metacaolín. Para las muestras M1, M2 y M3 se utilizó un recipiente de cuarzo dentro de otro metálico, sellado herméticamente (Ver Fig. 3a) y para las muestras M4, M5 y M6 se realizó un montaje de recirculación de agua, para condensar y retornar los vapores que se producían a una temperatura de $80^{\circ} \mathrm{C}$ (Ver Fig. 3b).

Fig. 3. MONTAJE METÁLICO HERMÉTICO Y DE RECIRCULACIÓN DE AGUA (A) Y CONDENSACIÓN DE LOS VAPORES LIBERADOS EN EL PROCESO (B)

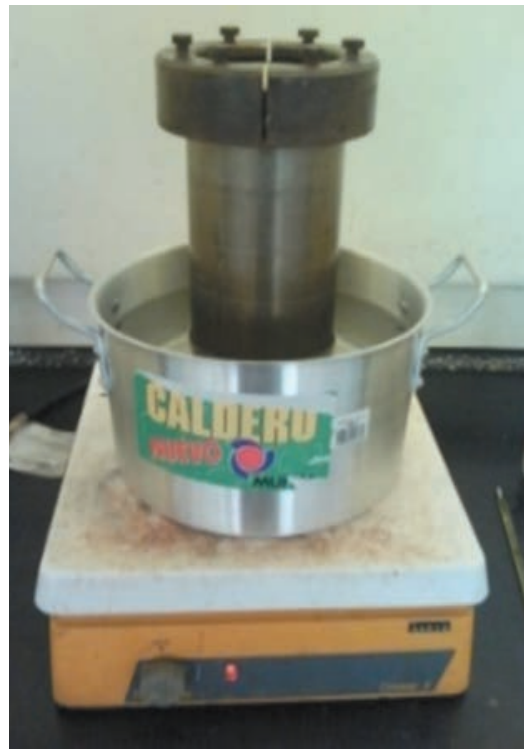

(A)

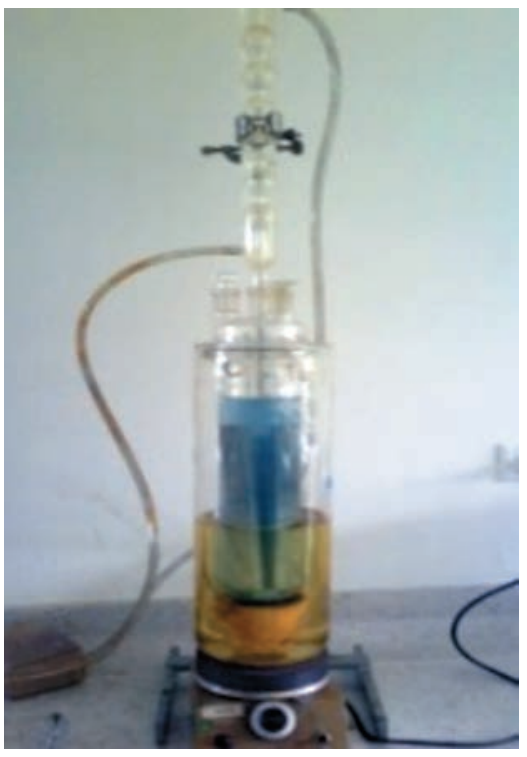

(B) 
Para medir la cantidad de luz (transmitancia) que podía pasar por el material de concreto modificado y traslúcido, se elaboraron 3 especímenes cúbicos de $5 \mathrm{~cm}$ de lado designados como M1, M2 y M3 (con adición de fibras ópticas), sometidas a un curado de 28 días. Para descubrirlas y obtener traslucidez, cada especimen se cortó $5 \mathrm{~mm}$ en cada uno de sus extremos. Estas muestras fueron expuestas a radiación directa de rayos láser (Rojo y Verde) y luz directa del sol, se introdujo cada una de las probetas en una caja de color negro para evitar pérdidas y enfocar mejor la luz que provenía de las fuentes. Las intensidades de los rayos incidentes (I) utilizados, se indican en la Tabla V.

TABLA V

INTENSIDAD DEL RAYO LÁSER

\begin{tabular}{|l|c|c|}
\hline Láser & \multicolumn{2}{|c|}{ Intensidad } \\
\hline Rojo & 7.84 & $\mu \mathrm{w}$ \\
\hline Verde & 7.89 & $\mu \mathrm{w}$ \\
\hline Solar & 19.2 & $\mathrm{mw}$ \\
\hline
\end{tabular}

La transmitancia de cada una de las muestras se obtuvo a partir de la ecuación (4).

$$
T=\frac{\mathrm{I}}{\mathrm{Io}} * 100
$$

Donde $I_{0}$ es la intensidad del rayo incidente e $I$ es la intensidad de la luz que viene de la muestra.

La capacidad del material para permitir el paso del espectro visible, se determinó mediante un espectrofotómetro de marca UV-2401PC. Para esto, se hizo incidir luz del espectro visible a cada una de las muestras (M1, M2 y M3) utilizadas en el ensayo de transmitancia, en un área de aproximadamente 1 $\mathrm{cm}^{2}$, durante 5 minutos.

\section{DISCUSIÓN DE RESULTADOS}

Los resultados que se presentan en las Fig. 4,5 y 6 denominados como probeta con fibra óptica corresponden a los obtenidos para un concreto modificado con $5 \%$ de fibra óptica y $83.3 \%$ de vidrio reciclado.

La Fig. 4 indica que la resistencia a la compresión del concreto sin contenido de fibras ópticas, a los 28 días, alcanzó $18.41 \mathrm{MPa}$; mientras que el concreto con fibras redujo esta resistencia un $20 \%$, obtenido un valor de $14.73 \mathrm{MPa}$.

La resistencia a la flexión del concreto sin fibras, a los 28 días fue de $3.83 \mathrm{MPa}$, valor que disminuyó un $14.36 \%$ para el concreto con adición de fibras ópticas, cuya resistencia obtenida fue de $3.28 \mathrm{MPa}$. Estos resultados se muestran en la Fig. 5 .
Los valores obtenidos de resistencia a la tracción a los 28 días, que se observan en la Fig. 6, fueron: 1.22 MPa para el concreto sin fibras ópticas y 1.05 MPa para el concreto con adición de fibras, valor 14 $\%$ menor.

El tipo de falla generada en cada una de las probetas ensayadas, corresponde a la característica para cada uno de los sistemas sometidos a esfuerzos de compresión, tracción y flexión.

Los ensayos de permeabilidad, indican que el material es más permeable, cuando el flujo pasa en dirección paralela a la ubicación de las fibras ópticas. Debido a que las fibras son continuas de extremo a extremo de la probeta, se generan capilaridades que hacen que sea más permeable, mientras que en el sentido transversal su permeabilidad disminuye un $60 \%$ ya que, existen menos espacios vacíos por donde pueda pasar el fluido. Los datos de permeabilidad se presentan en la Tabla VI.

TABLA VI

RESULTADOS ENSAYO PERMEABILIDAD

\begin{tabular}{|c|c|c|c|}
\hline Muestras & Permeabilidad & $\begin{array}{c}\text { Dirección a } \\
\text { las Fibras }\end{array}$ & $\begin{array}{c}\text { Perpendicular } \\
\text { a las Fibras }\end{array}$ \\
\hline M1 & $\mathrm{K}[\mathrm{Md}]$ & 9.59 & 4.48 \\
\hline M2 & $\mathrm{K}[\mathrm{Md}]$ & 11.75 & 3.71 \\
\hline M3 & $\mathrm{K}[\mathrm{Md}]$ & 11.96 & 5,24 \\
\hline
\end{tabular}

Los valores de porosidad obtenidos, resultan ser similares para todas las muestras ensayadas. Los resultados se muestran en la Tabla VII.

TABLA VII

RESULTADOS ENSAYO DE POROSIDAD

\begin{tabular}{|c|c|c|}
\hline Muestras & $\begin{array}{c}\text { Porosidad } \\
{[\%]}\end{array}$ & $\begin{array}{c}\text { \% Porosidad Promedio Probe- } \\
\text { ta de Comparación }\end{array}$ \\
\hline M1 & 21.85 & 16.82 \\
\hline M2 & 21.57 & \\
\hline M3 & 21.94 & \\
\hline
\end{tabular}

En el ensayo de reactividad álcali-agregado, se midió la expansión y deformación de cada una de las muestras con y sin adición de metacaolín (MK). Los especímenes sin adición de metacaolín (M1, M2 y M3), presentaron la mayor expansión y deformación, respecto a las muestras con adición de metacaolin (M4, M5 y M6), cuyas deformaciones se encuentran dentro del rango admisible especificado en la ASTM C1260. El porcentaje promedio de expansión para muestras sin adición de metacaolín (MK) es de $0.287 \%$ y para muestran con contenido de metacaolín (MK) es de $0.056 \%$. Los resultados del ensayo se muestran en la Fig. 7. 
Fig. 4. RESULTADOS DEL ENSAYO A COMPRESIÓN (IZQUIERDA) Y FALLA QUE SE PRESENTÓ EN LA PROBETA ENSAYADA (DERECHA)

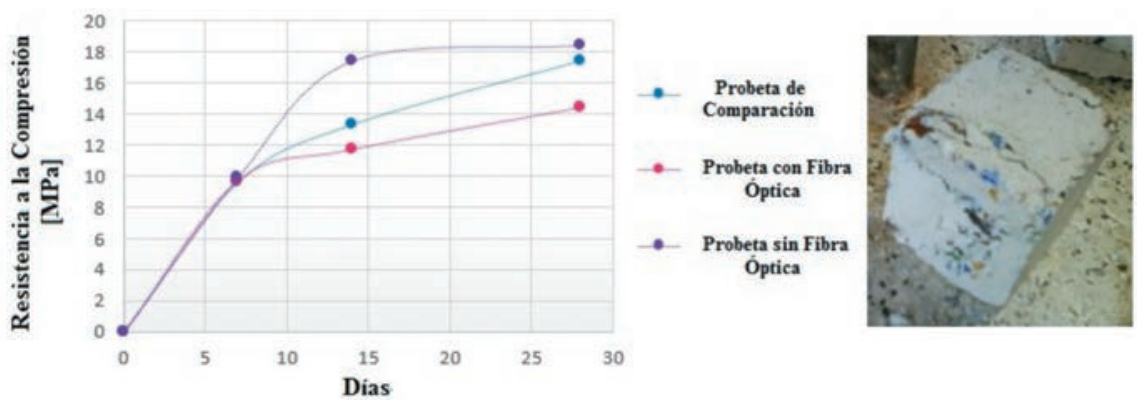

Fuente: Autores

FIG. 5. RESULTADOS DEL ENSAYO A FLEXIÓN (IZQUIERDA) Y FALLA QUE SE PRESENTÓ EN LA PROBETA ENSAYADA (DERECHA)

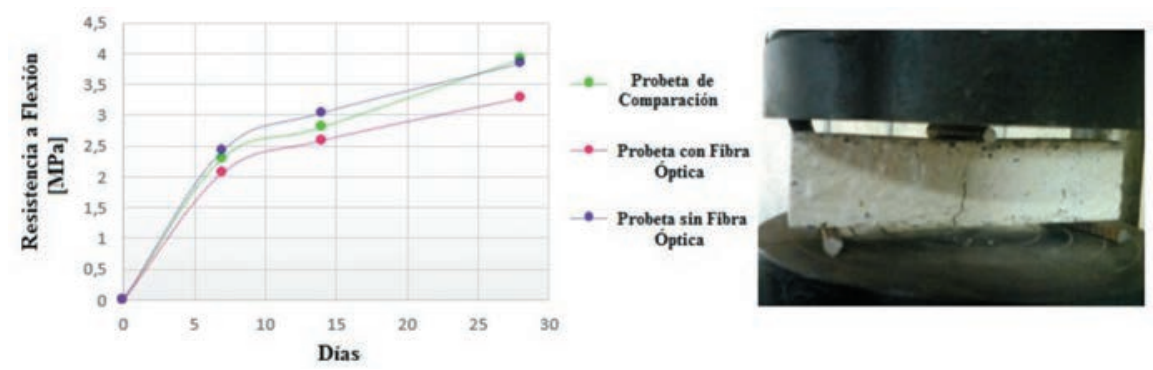

Fuente: Autores

Fig. 6. RESULTADOS DEL ENSAYO A TRACCIÓN (IZQUIERDA) Y FALLA QUE SE PRESENTÓ EN LA PROBETA ENSAYADA (DERECHA)

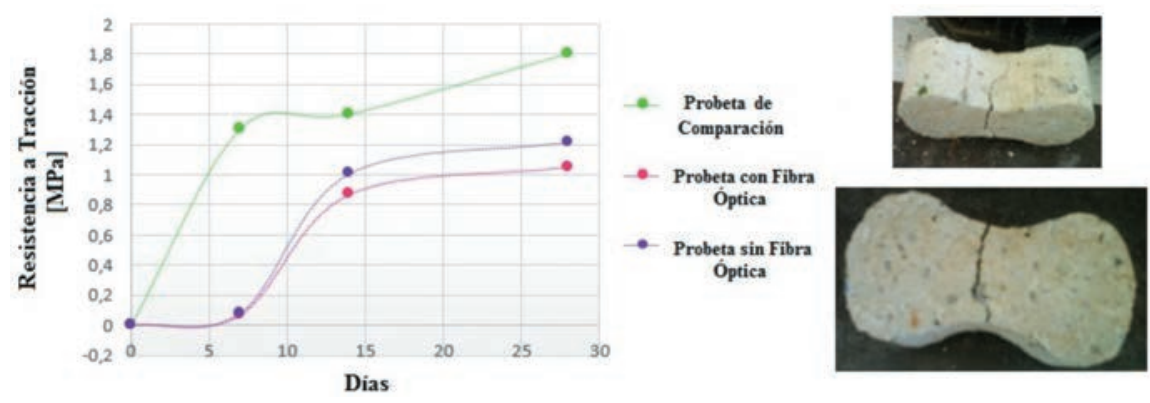

Fuente: Autores

FIG. 7. \% EXPANSIÓN DEL MATERIAL (IZQUIERDA) Y DEFORMACIONLAS MUESTRAS (DERECHA)
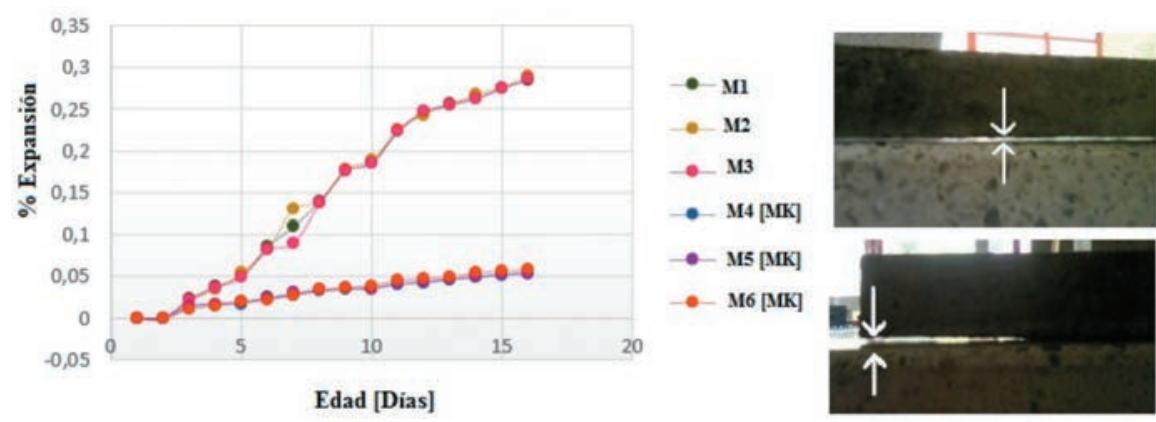

Fuente: Autores 
Los resultados obtenidos del ensayo de absorción capilar, indican que las muestras en contacto con agua, en su parte frontal (dirección paralela a las fibras ópticas) M1 y M2, se saturaron más rápido que aquellas en contacto con el agua, en su parte lateral (dirección perpendicular a las fibras ópticas), tal como se observa en la Fig. 8; siendo $t$ 0.5 la raíz cuadrada del tiempo.

Fig. 8. CANTIDAD DE AGUA ABSORBIDA EN FUNCIÓN DE LA RAíz CUADRADA DEL TIEMPO

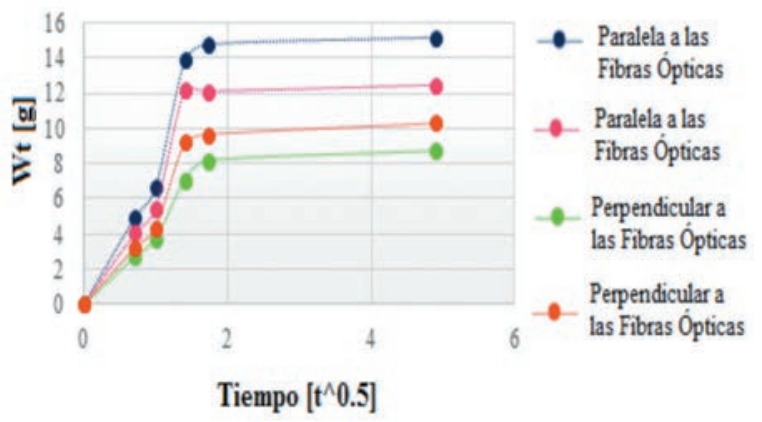

Fuente: Autores

TABLA VIII

CAPACIDAD DE ABSORCIÓN CAPILAR EN 24 HORAS

\begin{tabular}{|c|c|}
\hline Muestras & Capacidad de Absorción Capilar \\
\hline M1 & 1.79 \\
\hline M2 & 1.74 \\
\hline M3 & 1.69 \\
\hline M4 & 1.59 \\
\hline M5 & 0.2 \\
\hline
\end{tabular}

De la evaluación de la transmitancia, se encontraron valores menores al 1\% (Ver Fig. 9). La intensidad de la luz registrada por el fotómetro es muy pequeña; sin embargo, es posible observar que la luz pasa a través del material, tal como se evidencia en la Fig.10. A pesar de que la cantidad de luz que pasa a través de las muestras es muy pequeña, la capacidad del material para permitir el paso del espectro electromagnético es suficiente para que pase por completo.

En la Fig. 11 se aprecia las longitudes de onda registradas por el espectrofotómetro con y sin muestra.

\section{CONCLUSIONES}

Se realizó una dosificación apropiada de vidrio, metacaolín y fibra óptica, que permitió estabilizar la reacción álcali-agregado y obtener na mezcla de concreto con características de traslucidez.

De acuerdo a los resultados obtenidos de los ensayos de compresión, tracción y flexión, se dispone de un concreto modificado y adicionado para uso no estructural, con virtudes para la utilización de material reciclado. Se trata de un material homogéneo, con una porosidad cercana al $22 \%$.

A partir de los ensayos de permeabilidad y absorción capilar realizados, se concluye que este material es más susceptible a la acción de los fluidos en dirección paralela de las fibras ópticas, que en dirección perpendicular a éstas. La mayor permeabilidad en este sentido, puede deberse a que las fibras hacen las veces de capilares, ya que en toda su extensión se generan poros muy pequeños que se entrelazan y provocan este efecto. Similarmente sucede en el ensayo de absorción capilar, las fibras actuando como capilares hacen que el fluido ascienda por éstas más rápidamente.

Se evaluó la durabilidad del concreto mediante la adición de un material puzolánico como el metacaolín (MK), se observó cierta disminución en las expansiones provocadas por la reacción álcali-sílice, mejorando su resistencia frente a la acción de agentes externos que deterioran el material.

La capacidad de transmisión de luz del material, evaluada a partir de los ensayos de Transmitancia y Espectrofotometría, indican que la cantidad de luz que atraviesa las muestras es muy pequeña (menor al 1\%); sin embargo, se observa que es suficiente para que el ojo humano la capte. En este caso, el vidrio no hace ningún aporte a la translucidez, este sólo se utiliza como agregado para obtener una superficie agradable a la vista, es decir, solo como uso arquitectónico.

Se obtuvo un concreto no estructural de 14.73 MPa, no se logró la resistencia de diseño, posiblemente por la adición de fibras ópticas. Sin embargo, esta resistencia puede ser considerada alta para la funcionalidad del concreto, por lo que se recomienda para futuros estudios aumentar el porcentaje de adición de fibra óptica con el propósito de incrementar los niveles de transmitancia obtenidos.

\section{AGRADECIMIENTOS}

Los autores agradecen la participación y contribución de la Ingeniera Civil Karen Andrea Torrado Vergel en la realización de esta investigación. 
Fig. 9. CANTIDAD DE LUZ QUE TRANSMITEN LAS MUESTRAS (DERECHA) Y TRANSMITANCIA DE LAS MUESTRAS M1, M2 Y M3 (IZQUIERDA)
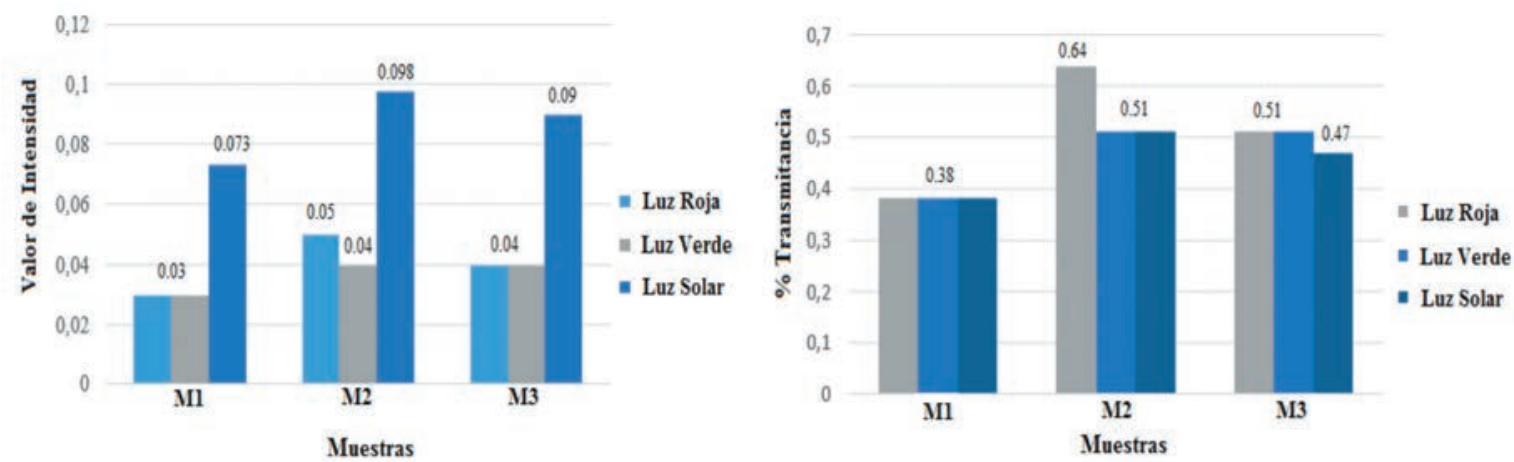

Fuente: Autores

FIG. 10. INTENSIDAD DEL RAYO LÁSER: ROJA (A) Y VERDE (B) Y LUZ SOLAR PROVENIENTE DE LAS MUESTRAS (C)

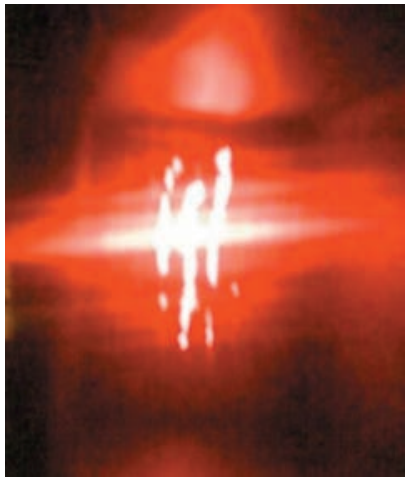

(A)

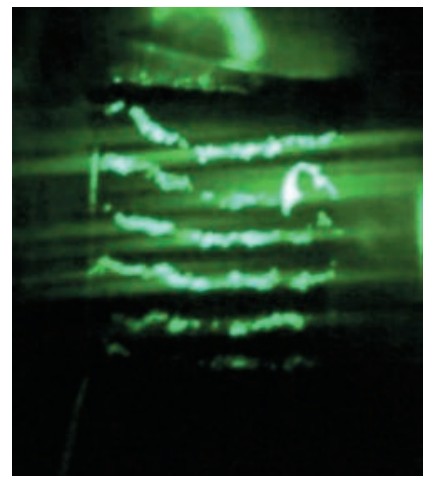

(B)

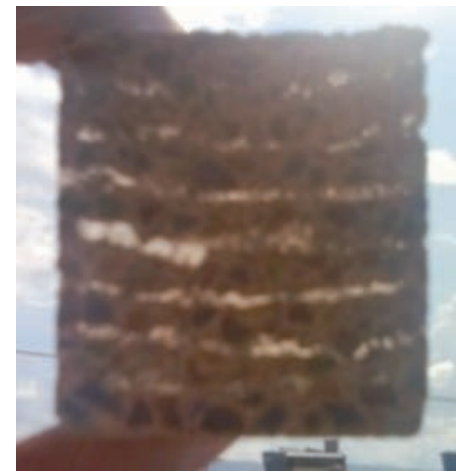

(C)

Fuente: Autores

Fig. 11. LONGITUD DE ONDA DEL ESPECTROFOTÓMETRO CON Y SIN LA MUESTRA 1 (IZQUIERDA) YDETALLE DE LA LONGITUD DE ONDA QUE REGISTRO LA MUESTRA 1 (DERECHA)
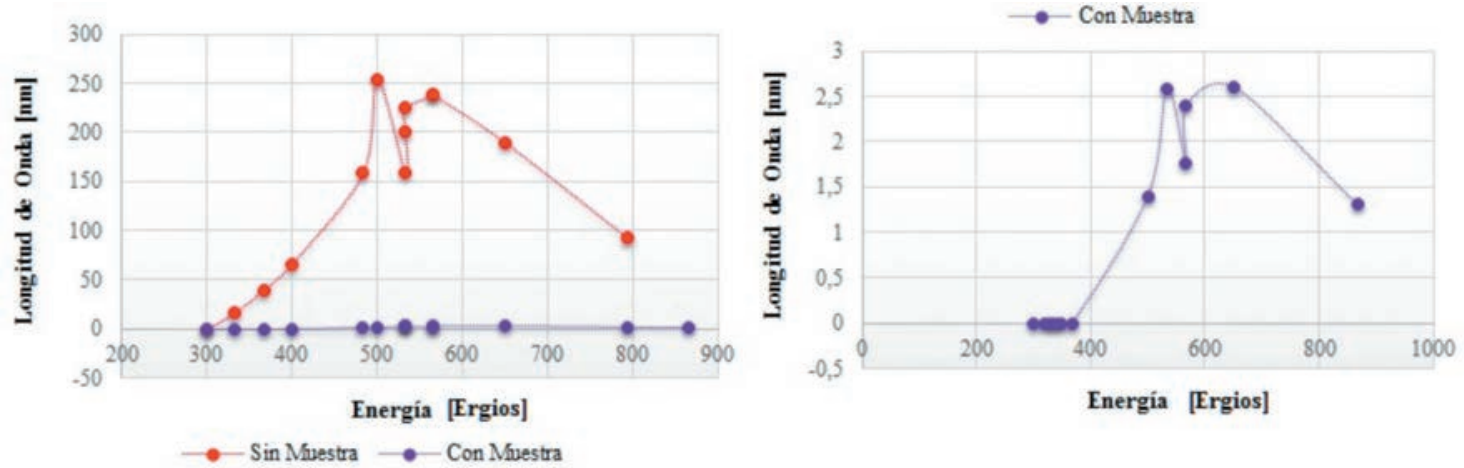

Fuente: Autores 


\section{REFERENCIAS}

[1] S. B. Park, B.C. Lee, J.H. Kim. "Studies on mechanical properties of concrete containing waste glass aggregate". Cement and Concrete Research No. 34 pp 2181-2189. 2004

[2] C. Shi, K. Zheng. "A review on the use of waste glasses in the production of cement and concrete". Resources Conservation and Recycling No. 52 pp 234-247. 2007

[3] R.K. Dhir, T.D. Dyer, M.C. Tang. "Alkali-silica reaction in concrete containing glass". Materials and Structures No. 42 pp 1451-1462. 2009

[4] R. Siddique, J. Klaus. "Influence of metakaolin on the properties of mortar and concrete: A review". Applied Clay Science No. 43 pp 392-400. 2009

[5] Z. Zhou, G.Ou, Y. Hang, G. Chen, J. Ou. "Research and development of plastic optical fiber based smart transparent concrete", Proc. of SPIE Vol. 7293 72930F-1.

[6] T. Ramlochan, M. Thomas, K.A. Gruber. "The effect of metakaolin on alkali-silica reaction in concrete". Cement and Concrete Research No. 30 pp 339-344. 2000

[7] H. Maraghechi, S.Shafaatiana, G.Fischer, F. Rajabipour. "The role of residual cracks on alkali silica reactivity of recycled glass aggregates". Cement \& Concrete Composites 34 pp 41-47. 2012

[8] D.F. Arciniegas López, C. Fonseca. “Utilización de Vidrio Reciclado en Morteros". Tesis de Grado. Universidad Industrial de Santander, Colombia. 2004

[9] Norma Técnica Colombiana, NTC 220. Determinación de la resistencia de morteros de cemento hidráulico usando cubos de $50 \mathrm{~mm}$ ó 50,8 mm de lado.
[10] Norma Técnica Colombiana, NTC 120. Método de ensayo para determinar la resistencia a flexión de morteros de cemento hidráulico.

[11] Norma Técnica Colombiana, NTC 119. Determinación de la Resistencia a la tensión de morteros de cemento hidráulico.

[12] Society of Engineers and Architects, SIA 162. Swiss Standar - SucciónCapilar.

[13] American Society for Testing and Materials, ASTM C1260. 2007. Standard Test Method for Potential Alkali Reactivity of Aggregates (Mortar-Bar Method).

[14] American Society for Testing and Materials, ASTM C490 / C490M. 2011. Standard Practice for Use of Apparatus for the Determination of Length Change of Hardened Cement Paste, Mortar, and Concrete.

[15] O. Karahan, K.M.A. Hossain, E. Ozbay, M. Lachemi, E. Sancak. "Effect of metakaolin content on the properties self-consolidating lightweight concrete". Construction and Building Materials No. 31 pp 320-325. 2012

[16] A.G. Mainini, T. Poli, M. Zinzi, S. Cangiano. "Spectral light transmission measure and radiance model validation of an innovative transparent concrete panel for façades". Energy Procedia No. 30 pp. 1184 - 1194. 2012

[17] K. Shi-Cong, P. Chi-Sun. "A novel polymer concrete made with recycled glass aggregates, fly ash and metakaolin". Construction and Building Materials No. 41 pp 146-151. 2013 